\title{
A vitamina A no processo inflamatório da acne: avaliação do uso da farinha de cenoura como alternativa na redução da inflamação
}

\author{
Vitamin A in the inflammatory process of acne: evaluation of the use of \\ carrot meal as an alternative in the reduction of inflammation
}

\author{
Carolina Gomes de Alcântara, \\ Yana Maria Amaral Rocha', \\ Mônica Gouvea de Oliveira1, \\ Gabriela Soares dos Santos ${ }^{1}$, \\ EmiliRafaeli de Faria ${ }^{1}$, \\ Gilce Santos Olivera ${ }^{1}$, \\ Brenda Ferraz Vieira ${ }^{1}$ \\ Michelle Rosa Andrade Alves², \\ Raquel Linhares Bello de Araújo³, \\ Mauro Ramalho Silva ${ }^{3}$, \\ William César Bento Regis ${ }^{4}$
}

Artigo recebido em 25 de Setembro de 2017 e aprovado em 26 de Novembro de 2018

\footnotetext{
${ }^{1}$ Acadêmicas do curso de Nutrição da Pontifícia Universidade Católica de Minas Gerais- PUCMG. Brasil. Email: carolinaalcantara.2@gmail.com; yanarocha20@gmail.com; monicagouvea_ol@yahoo.com.br; gabiela.soaresdossantos@gmail.com; emili.rafaeli@yahoo.com; oliveira.gilceoliveira@gmail.com; brendaferraz96@gmail.com

2 Professora Adjunta de Nutrição da Pontifícia Universidade Católica de Minas Gerais- PUCMG. Brasil. Email: michele.alves@gmail.com

${ }^{3}$ Programa de Pós-Graduação em Ciência de Alimentos na Universidade Federal de Minas Gerais- UFMG. Brasil. Email: mauroramalhosilva@ yahoo.com.br;

4 Programa de Pós-Graduação em Nutrição e Saúde, Programa de Pós- Graduação em Biologia de Vertebrados Universidade Federal de Minas Gerais-UFMG e Programa de Pós- Graduação em Infectologia e Medicina Tropical, pela Pontifícia Universidade Católica de Minas Gerais- PUCMG. Brasil. E-mail: wcbregis@gmail.com
} 


\begin{abstract}
RESUMO
Introdução: A acne é uma doença inflamatória crônica que atinge de 85 a $100 \%$ da população e tem maior ocorrência na adolescência. O quadro inflamatório da acne pode ser reduzido com a inserção de vitamina A na dieta, já que a mesma age na inibição da indução da IL-17 pela bactéria Propionibacterium acnes. Entretanto, uma das principais fontes de vitamina A são os vegetais e o consumo dos mesmos é muito baixo na população brasileira. Objetivo:O presente estudo teve como objetivo elencar os benefícios, cientificamente comprovados em humanos da vitamina A, visando criar um painel de potencialidades de seu uso por meio de novas formas de introdução do nutriente na alimentação do brasileiro. Metodologia: a) foi realizada uma revisão sistemática da literatura no PubMed/MedLine, com restrição para ensaios clínicos randomizados em humanos; b) foi produzida, por secagem em estufa, uma farinha de cenoura; c) foi realizada a determinação de carotenoides totais para comparação com outras farinhas existentes no mercado; d) foi produzida uma massa, tipo macarrão, enriquecida com a farinha e a mesma; e) foi conduzida a análise sensorial com teste afetivo aplicado a 110 indivíduos, escolhidos de forma aleatória. Resultados: a) foram encontrados 851 artigos e após aplicação dos critérios de inclusão e exclusão (humanos, sem associação medicamentosa) foram selecionados os artigos com melhor nível de evidência para ilustração dos efeitos da vitamina A; b) a farinha produzida apresentou $8 \%$ de umidade, aspectos de coloração e textura normais comparado com padrões comerciais; c) a farinha de cenoura produzida apresentou $106,7 \mathrm{mg} / 100 \mathrm{~g}$ de carotenoides totais, o que corresponde rendimento superior, de 79,6 a 95,3\%, comparado com outras farinhas disponíveis no mercado; d) a massa elaborada apresentou textura e aspectos gerais de manipulação e cocção compatíveis com outras massas; e) a análise sensorial obteve $81,81 \%$ de aceitação positiva em relação a aparência, odor, sabor e textura. Conclusão: Estudos com elevado nível de evidência científica tem comprovado a efetividade da vitamina A nas complicações da acnee a farinha de cenoura produzida neste estudo apresentou-se como excelente fonte de carotenoides, além de demonstrar excelente potencial de uso em preparações com melhor aceitação de consumo entre a população brasileira, consequentemente, reduzindo o processo inflamatório da acne.
\end{abstract}

Palavras-Chave: Vitamina A, Antioxidantes, Acne

\begin{abstract}
Introduction: Acne is a chronic inflammatory disease that affects 85 to $100 \%$ of the population and has a higher occurrence in adolescence. The inflammatory picture of acne can be reduced with the insertion of vitamin A in the diet since it acts in inhibition of the induction of IL-17 by Propionibacterium acnes. However, vegetable consumption, sources of vitamin A, is very low in the Brazilian population. Objective: The objective of this study was to list the scientifically proven benefits of vitamin A to create a panel of potentials for its use through new ways of introducing the nutrient into the Brazilian diet. Methodology: a) a systematic review of the literature was performed in PubMed / MedLine, with restriction for randomized clinical trials in humans; b) a carrot meal was produced by drying in a greenhouse; c) total carotenoids were determined for comparison with other flours on the market; d) one of pasta-like pasta enriched with flour was produced; e) the analysis was carried out with affective test applied to 110 individuals, randomly chosen. Results: a) were found 851 articles and after applying the inclusion and exclusion criteria (human, without drug association) were selected the articles with the best level of evidence to illustrate the effects of vitamin $\mathrm{A} ; \mathrm{b}$ ) the flour produced presented $8 \%$ moisture, normal staining and texture aspects compared to commercial standards; c) the produced carrot meal had $106.7 \mathrm{mg} / 100 \mathrm{~g}$ of total carotenoids, which corresponds to a higher yield, from 79.6 to $95.3 \%$, compared to other flours available on the market; d) the elaborated dough presented texture general aspects of handling and cooking compatible with other masses; e) sensory analysis obtained $81.81 \%$ positive acceptance regarding appearance, odor, taste and texture. Conclusion: Studies with a high level of scientific evidence have demonstrated the effectiveness of vitamin $\mathrm{A}$ in the complications of acne and the carrot meal produced in this study presented as an excellent source of carotenoids, as well as showing excellent potential of use in preparations with better acceptance of consumption for the Brazilian population.
\end{abstract}

Key Words: Vitamin A, Antioxidants, Acne 


\section{Introdução}

A acne é uma doença cutânea que afeta de 85 a 100\% da população em algum momento da vida, iniciando geralmente na puberdade, com pico de incidência em adolescentes entre 14 e 17 anos e nos indivíduos do sexo masculino entre 16 e 19 anos (AGAK et al., 2014). A acne é caracterizada como uma doença inflamatória crônica que ocorre em locais da pele com alta densidade de glândulas sebáceas (NAST et al., 2012). Tem habitualmente um desconforto físico e também psicológico de curto prazo, mas com potencialidade de se manter e tornar-se grave, levando ao afastamento social e mesmo a depressão, e, quando não tratada pode dar origem a cicatrizes inestéticas ou mesmo desfigurantes (FIGUEIREDO et al., 2011).

Segundo Kurokawa et al. (2009), acredita-se que vários fatores primários e secundários contribuem para o aparecimento e desenvolvimento da acne, mas o principal fator fisiopatológico é, provavelmente, um aumento induzido por andrógenos na produção de sebo e secreção. As características na composição do sebo em níveis reduzidos de ácido linoleico, níveis aumentados de esqualeno e peróxidos lipídicos e uma maior proporção de ácidos graxos saturados e monoinsaturados (ZOUBOULIS et al., 2014). Na sua formação, há uma hipertrofia de toda a glândula decorrente da ação androgênica sobre sua estrutura, criando condições para a formação do comedão que pode evoluir para inflamação e lesões inflamatórias como pápula, pústula e nódulos. São fatores contribuintes para o desenvolvimento da acne: ${ }^{1}$ Hiperplasia Sebácea; ${ }^{2}$ Diferenciação Folicular Anormal; ${ }^{3}$ Colonização por Propionibacterium acnes; Inflamação. ${ }^{4}$

A hiperplasia sebácea ocorre sob influência de hormônios androgênios que provocam aumento da taxa de secreção sebácea. A diferenciação folicular anormal causa obstrução do ducto polissebáceo e bactéria anaeróbia Propionibacterium acnes desempenha um papel importante na patogênese da acne, pois causa uma inflamação que desencadeia uma resposta imunológica no organismo do hospedeiro que libera citocinas inflamatórias (AGAK et al., 2014).

\footnotetext{
${ }^{1}$ Hiperplasia Sebácea: aumento no tamanho das glândulas sebáceas, produção de sebo.

${ }^{2}$ Diferenciação Folicular anormal: alteração do crescimento do folículo (hiperqueratinização)

${ }^{3}$ Colonização por Propionibacterium Acnes: microorganismos isolados da pele

${ }^{4}$ Inflamação: produção de lipases e fosfatases, neutrófilos e linfócitos estimulando produção de IL-8, IL-1 $\beta$ (RIBEIRO et al., 2015)
} 
Níveis adequados de vitamina A auxiliam na melhora do processo inflamatório da acne. De acordo com Beckenbach (2015), a vitamina A afeta as células humanas nos estágios de embriogênese, reprodução, regulação do processo inflamatório, crescimento celular e diferenciação.

A vitamina A foi descoberta por volta de 1906 e sintetizada em meados de 1947 e pode ser encontrada em duas formas principais, carotenoides e retinóis, nos alimentos (CHAPMAN, 2012). O retinóide, uma forma de vitamina A, é necessário para corrigir a queratinização em doenças de pele, tendo efeito inibitório altamente eficaz sobre as glândulas e produção de sebo, o que leva a redução da população de Propionibacterium acne, que é um dos fatores etiogênicos.

Segundo Bieski (2016), a isotretinoina (figura 1) é um retinóide encontrado em organismos de origem animal e vegetal como carotenoide, é encontrado em vegetais verdeescuros e legumes alaranjados e seu principal mecanismo de ação ocorre na glândula sebácea, reduzindo seu tamanho, atividade e quantidade de sebo produzido em até $75 \%$, após quatro semanas de tratamento. A vitamina A age na inibição da indução da IL-17 por Propionibacterium acnes, reduzindo o número de células Th17 já que estas são produtoras de IL-17 na patogênese da acne (AGAK, 2014). São diversas as fontes de vitamina A, entretanto, segundo Chapman (2012), em 100g de cenoura contém cerca de $93 \%$ da média diária recomendada de beta-caroteno.

\section{Figura 1: Ácido 13 -cis-retinóico}

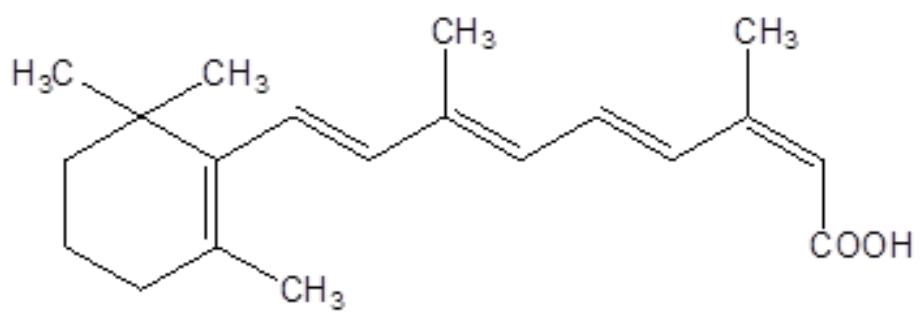

Segundo Medeiros et al. (2011), a cenoura, Daucus carota L. é uma raiz com grande versatilidade para ser industrializada e transformada em diversos produtos 
destinados à alimentação humana. Portanto, o presente estudo teve como objetivo elencar os benefícios da vitamina A presente na farinha de cenoura por meio de revisão bibliográfica, visando criar um painel de potencialidades de seu uso na prevenção, tratamento e redução da acne, além de avaliar a qualidade nutricional das farinhas disponíveis no mercado e compará-las a farinha produzida no estudo através de análise bromatológica. Também foi preparada uma massa com a farinha obtida e avaliou-se a composição centesimal da mesma no qual os nutrientes foram calculados através dos dados da TACO (tabela de composição de alimentos).

\section{METODOLOGIA}

\section{Análise de Literatura Técnica}

Foi realizada uma revisão bibliográfica sobre a importância e a influência da Vitamina A na patogênese da acne e seu processo de inflamação. A revisão foi dividida em duas etapas: a primeira consiste na procura de artigos por meio do PubMed/MedLine usando os descritores "acne" e "vitamin A" encontrando um número de 851 resultados. Após a busca foi feita a leitura do título e resumo observando critérios de inclusão e exclusão. Os critérios de inclusão foram: artigos dos últimos 10 anos, estudos com uso de vitamina A na forma oral e ensaios clínicos avaliados em humanos. Os estudos foram expressamente limitados a ensaios com humanos, trazendo níveis de evidência entre $1 \mathrm{~B}$ e 1C, consideráveis para tornar a revisão íntegra e concreta. Como critérios de inclusão foram escolhidos artigos que relataram ingestão oral da vitamina, para avaliar e comparar com melhor precisão o efeito de absorção da vitamina A, ora por meio de cápsulas farmacêuticas ora por meio da ingestão dietética.

A segunda etapa consistiu na leitura completa dos artigos, sendo descartados os estudos que não relacionavam o uso oral da vitamina A com seu efeito na função imunológica da inflamação na acne. Os critérios de exclusão foram: artigos anteriores a 2006; estudos analisados em animais; uso do retinoide na forma de creme, gel, pomada ou equivalentes; uso comparativo da isotretinoína com adapaleno e afins. Na figura 2 está exposto a descrição e seleção da pesquisa. 


\section{Figura 2 - Fluxograma de descrição e seleção da pesquisa de dados}

Publicações identificadas

por meio da pesquisa de dados (n: 851)

Publicações com texto completo para avaliar (n: 64)

Publicações incluídas

(n: 4)
Publicações excluídas por critérios de exclusão ( $n: 787$ )

Publicações excluídas por falta de correlação (n: 60)

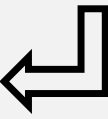

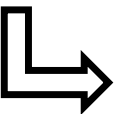
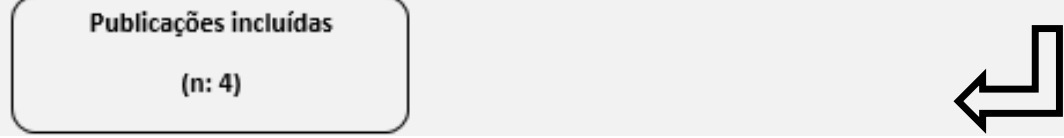

\section{Produção da Farinha e produto}

Cenouras (Daucus carota), foram adquiridas no comércio local da cidade de Belo Horizonte, MG-Brasil. As amostras foram levadas ao laboratório de bromatologia da Pontifícia Universidade Católica de Minas Gerais - PUC MINAS, unidade barreiro para as etapas de: seleção, classificação, lavagem em água potável, sanitização e secagem.

A limpeza da amostra foi realizada retirando-se as sujidades mais grosseiras como: terra e poeira, sob água corrente e potável. Após serem previamente lavadas, as cenouras passaram por processo de sanitização, conforme as recomendações da Portaria CVS - 6/99, de 10 de março de 1999.

\section{Preparação da Farinha}

As cenouras, aproximadamente $1 \mathrm{~kg}$, foram raladas em ralo de $2 \mathrm{~mm}$, pesadas, distribuídas uniformemente em bandejas e submetidas à secagem artificial em Estufa para Esterilização e Secagem, modelo 400/D $300^{\circ} \mathrm{C}$, com circulação forçada de ar, empregando a temperatura de $50^{\circ} \mathrm{C}$ por um período de 24 horas. Depois de seca, a amostra foi 
processada em liquidificador/processador doméstico Philips Walita 500W, modelo RI 2035, até a obtenção de uma farinha homogênea e, então, peneirada. Foram produzidas $100 \mathrm{~g}$ de farinha, que foi armazenada em ambiente isento de luz, devido sua oxidação, até a preparação da massa para análise sensorial da mesma.

\section{Análise Bromatológica}

Foram analisadas três amostras de farinha de cenoura no laboratório de bromatologia da PUC MINAS- Pontifícia Universidade Católica de Minas Gerias: farinha A, farinha B e a farinha $\mathrm{P}$ (produzida). As farinhas A e B foram adquiridas no Mercado Central de Belo Horizonte, por meio de escolha aleatória, no qual as farinhas A e B foram as primeiras encontradas no comércio local, assim sendo selecionadas para avaliação e comparação do estudo.

Foram avaliados os carotenoides totais das farinhas por meio da determinação de carotenoides totais, conforme procedimento descrito por Rodriguez-Amaya et al. (1976). Os procedimentos para determinação de carotenoides, iniciando-se a partir da extração das amostras foram determinados cuidadosamente, levando-se em conta a necessidade de proteger da incidência de luz difusa.

Foram pesados 0,5g das amostras de farinhas de cenoura em tubo tipo Falcon de 50 $\mathrm{mL}$ e acrescentado juntamente à amostra, 0,05g de Celite. As amostras foram adicionadas de $25 \mathrm{~mL}$ de acetona e homogeneizadas a $13.000 \mathrm{rpm}$ por 1 minuto em ultra-turrax. Em seguida, foram centrifugadas a $263 \mathrm{xg}$ (força centrifuga) por 3 minutos e o sobrenadante foi retirado e transferido para outro tubo. O procedimento de extração com $25 \mathrm{~mL}$ de acetona e centrifugação foi repetido por mais duas vezes, até a amostra não apresentar a coloração alaranjada, característica dos carotenoides.

O extrato combinado foi transferido, quantitativamente, para um funil de separação, ao qual foi adicionado 40mL de hexano. Em seguida, três porções sucessivas de $150 \mathrm{~mL}$ de água deionizada, foram adicionadas e, posteriormente, drenadas a cada adição juntamente com a acetona. A porção de hexano, contendo os carotenoides extraídos foi filtrada através de lã de vidro e sulfato de sódio anidro para remoção da água remanescente. O extrato final foi transferido para um balão de $50 \mathrm{~mL}$ e o volume foi completado com hexano. 
A curva de calibração foi realizada com seis níveis de betacarotenoides de concentração, e nos seis níveis foram realizadas triplicatas. Extratos de amostras e soluções padrão da curva de calibração foram submetidos às leituras em espectrofotômetro a 450 $\mathrm{nm}$.

\section{Preparação da Massa}

Foi elaborada uma massa, tipo macarrão, enriquecida com a farinha de cenoura produzida. Segundo a resolução RDC $N^{\circ} 93$ de 31 de dezembro de 2000, a massa alimentícia ou macarrão, é um produto não fermentado, apresentado de várias formas, recheado ou não, obtido pelo empasto, amassamento mecânico de farinha de trigo comum e ou sêmola/semolina de trigo e ou farinha de trigo integral e ou farinha de trigo durum e ou sêmola/semolina de trigo durum e ou farinha de trigo integral durum, e ou não de temperos e ou complementos, isoladamente ou acondicionados diretamente à massa (ANVISA, 2000). Para preparo da massa, misturaram-se com espátula todos os ingredientes (farinha de cenoura, farinha de arroz, farinha de Banana Verde Ovos, Água, Sal, Clara de Ovo) em uma tigela. A massa foi misturada até adquirir consistência para sovagem com as mãos.

A massa foi sovada acrescentando-se água aos poucos até ficar homogênea. A massa foi aberta, cortada e reservada para o momento de cozimento e finalização da preparação.

\section{RESULTADOS E DISCUSSÃO}

\section{Análise de Literatura Técnica}

No PubMed foram encontrados 851 artigos com base nos descritores utilizados, dos quais 64 artigos atendiam aos critérios de inclusão. A partir da leitura foram excluídos 60 artigos por não apresentarem interação Vitamina A versus Inflamação da acne. Atingiram os objetivos do trabalho apenas quatro estudos, no qual dois ressaltavam a importância da vitamina A na dieta dos indivíduos para menor gravidade do quadro de acne e dois artigos citavam a influência e o poder da vitamina A agindo como imunomoduladora na inflamação da acne. O quadro 1 mostra o resumo dos estudos utilizados e analisados na pesquisa. 


\begin{tabular}{|c|c|c|c|c|c|c|c|}
\hline AUTOR E ANO & $\begin{array}{c}\text { MCOELO } \\
\text { DP. }\end{array}$ & $N^{2}$ INDIVDUOS & CEIETINOS & $\begin{array}{c}\text { Resul tados no } \\
\text { Drocesso inf am atório }\end{array}$ & CONCLUSÖES & OBSERVAÇŌES & $\begin{array}{l}\text { NIVEL DE } \\
\text { EVDÉNCIA }\end{array}$ \\
\hline $\begin{array}{c}\text { Oruguz p et of } \\
2014\end{array}$ & Humanos & $94 \mathrm{P}$ xientes & $\begin{array}{c}\text { Medir concenvaçôes } \\
\text { de vit. A plasmáticas } \\
\text { em relaçäo aos niveis } \\
\text { de gravidade de acne } \\
\text { do individuo }\end{array}$ & - & $\begin{array}{l}\text { pode se evideociar que } \\
\text { quanto maior o nivei de } \\
\text { gravidade da inflamação, } \\
\text { menor o nivel de vit. A } \\
\text { pasmatica do individuo }\end{array}$ & $\begin{array}{l}\text { O uso de retinoides } \\
\text { esta diretamente } \\
\text { associado a red uçào } \\
\text { da gravidade da } \\
\text { infl amaçào da acne }\end{array}$ & $\begin{array}{c}18 \\
\text { Grau de } \\
\text { Recomendação A }\end{array}$ \\
\hline $\begin{array}{c}\text { E-Akawi } z \text { et } \\
\text { of, } 2005\end{array}$ & Humanos & $\begin{array}{c}200 \text { Pacientes } \\
\text { Grupo controle: } 100 \\
\text { Grupo tratado: } 100\end{array}$ & $\begin{array}{c}\text { Medir concentrações } \\
\text { de Vir. A plasmaticas } \\
\text { por cromatografia } \\
\text { liquida }\end{array}$ & $\begin{array}{l}\text { Niveis reduzidos de } \\
\text { Vit. A aumentam os } \\
\text { niveis de metabolitos } \\
\text { toxicos de oxizenio e } \\
\text { geram acumul o de } \\
\text { neutrofilos no tecido } \\
\text { afetado }\end{array}$ & $\begin{array}{l}\text { Há uma forte relacio } \\
\text { entre a reduçào dos } \\
\text { niveis de Vit. A eo } \\
\text { aumento da gravidade } \\
\text { da coondiçào da acne }\end{array}$ & $\begin{array}{l}\text { vitamina A tem papel } \\
\text { importante na } \\
\text { patogenese da acne já } \\
\text { que esta em nive's } \\
\text { baixos aumenta a } \\
\text { gravidade da mesma }\end{array}$ & $\begin{array}{c}\text { IB } \\
\text { Grau de } \\
\text { Recomendaçio A }\end{array}$ \\
\hline $\begin{array}{l}\text { Siniavski luA } \\
\text { et al, } 2014\end{array}$ & Humanos & $\begin{array}{c}180 \text { Padientes } \\
\text { Grupo controle: } 90 \\
\text { Grupo com acne: } 90\end{array}$ & $\begin{array}{l}\text { Revelar infuêncis da } \\
\text { ingestão dietetica } \\
\text { sobre a gravidade da } \\
\text { acne, inclunindo a vit. } \\
\text { A }\end{array}$ & - & $\begin{array}{c}\text { Faita de vit. A (retino]) } \\
\text { sua provitamina } \\
\text { [Caroteno) afetam } \\
\text { signiffcativamentea } \\
\text { gravidade da acne }\end{array}$ & $\begin{array}{c}\text { Os micronutrientes } \\
\text { tem um gande poder } \\
\text { de influência sobre o } \\
\text { proce sso patologico } \\
\text { da acne }\end{array}$ & $\begin{array}{c}1 C \\
\text { Grau de } \\
\text { Recomendaçàio A }\end{array}$ \\
\hline $\begin{array}{l}\text { George W. } \\
\text { Agak et ol, } \\
2014\end{array}$ & mumanos & - & $\begin{array}{c}\text { Papel da vit. A na } \\
\text { regulaçio de celulas } \\
\text { Th17 }\end{array}$ & $\begin{array}{c}\text { Reduçào de } 4-17, e \\
\text { consequente reduç̄o } \\
\text { de Th17 produzid as } \\
\text { por }\{-17\end{array}$ & \begin{tabular}{|c|} 
Vit. A possui efeito \\
inibto fio na induç3o de \\
1L-17, sendo importante \\
para otratamento da \\
acne
\end{tabular} & $\begin{array}{c}\text { A ll-1 } 7 \text { produzem as } \\
\text { celulat Th17 que tem } \\
\text { um papel importante } \\
\text { na parogenese da } \\
\text { acne }\end{array}$ & $\begin{array}{c}\text { AB } \\
\text { Grau de } \\
\text { Recomendaça A }\end{array}$ \\
\hline
\end{tabular}

No estudo de Ozuguz et al. (2014), observou-se a evidência de que quanto maior o nível de gravidade da inflamação, menores serão os níveis plasmáticos de Vit. A no indivíduo. No estudo, os níveis plasmáticos de Vit. A do grupo controle (sem diagnóstico de acne) apresentaram-se bem mais elevados que os do grupo 1 (acne moderada) e grupo 2 (acne grave).

El-Akawi et al. (2006) mediram as concentrações plasmáticas de Vit. A em pacientes com acne, por meio do processo de cromatografia líquida, método físico-químico de separação dos componentes de uma mistura, realizada através da distribuição entre duas fases. Uma das fases permanece estacionária enquanto a outra move-se através dela. Durante o desenvolvimento, os componentes da mistura são distribuídos entre as duas fases resultando em migrações diferenciais destes componentes (TONHL. E, 2002). Assim observaram uma grande relação entre a redução dos níveis plasmáticos de Vit. A e o aumento da gravidade da condição da acne, isto porque quando os níveis de Vit. A estão baixos, existe um aumento dos níveis de metabolitos tóxicos gerando acúmulo de neutrófilos no tecido afetado. Os neutrófilos representam a linha de defesa do corpo contra patógenos através da fagocitose na liberação de quimiocinas e citocinas TNF-alfa e IL-1 (BORREGAARD et al., 2007), com o aumento dos mesmos a inflamação aumenta já que o organismo quer expulsar o patógeno, agravando a condição da acne. 
Siniavskii et al. (2014) estudaram 180 pacientes para revelar a influência da ingestão dietética de carboidratos, e micronutrientes, e entre eles a vitamina A na gravidade da acne. A baixa ingestão de vitamina A na dieta, na forma de retinol e de sua provitamina caroteno, afetam significativamente a gravidade da acne por apresentar resposta imune frente a inflamação.

O estudo de Agak et al. (2014) evidenciou o papel da vitamina A na regulação das células Th17. Pela análise de pele congelada dos pacientes estudados observaram que a presença da vitamina A, criou um efeito inibitório na indução de IL-17 e com isso redução da produção de células Th17. A IL-17 é uma interleucina que libera os neutrófilos para a área afetada, e se ela não está sendo induzida, há um menor número de Th17 e neutrófilos migrando para a área de inflamação da acne, reduzindo assim, o nível de gravidade da inflamação.

Dados apresentados pelo IBGE (2013) demonstraram que grande parte da população brasileira consumia frutas e verduras em uma porcentagem menor que a recomendada, e que sabidamente são fontes primárias de vitamina A sob a forma de betacaroteno, portanto, possivelmente parte desta população pode apresentar hipovitaminose A, que já é um problema de saúde pública no Brasil. Diversos estudos científicos evidenciam os benefícios, influência e importância da vitamina A na dieta para melhora do quadro de acne e interferência no processo de inflamação pelo seu poder imunomodulador. Desta forma, fica evidente a importância da introdução de alimentos ricos em vitamina $\mathrm{A}$, para garantir a equilibrada dieta da população e redução do quadro de hipovitaminose A, além de intervir sobre o processo inflamatório da acne.

\section{Análise Bromatológica}

Na Tabela 1 é possível notar que a quantidade de carotenoides totais presentes na farinha produzida é, indiscutivelmente, maior que nas demais farinhas. As farinhas A e B, já comercializadas, apresentaram 4,43mg e 19,01mg de carotenoides totais em $100 \mathrm{~g}$ de farinha respectivamente. A farinha produzida (FP) no laboratório pela secagem na estufa apresentou 106,07mg de carotenoides totais em $100 \mathrm{~g}$ de farinha. A farinha produzida apresentou melhores aspectos nutricionais e o processo manual de secagem na estufa 
considerou o fator de perda oxidativa da cenoura, levando em conta seu alto índice de perda de betacaroteno em altas temperaturas.

\section{Tabela 1: Composição centesimal Macarrão enriquecido com farinha de cenoura}

\begin{tabular}{cccc}
\hline Amostras & $\begin{array}{c}\text { Carotenoides totais } \\
(\mathbf{m g} / \mathbf{1 0 0 g})\end{array}$ & Desvio Padrão & \% CV \\
\hline Farinha A & 4,43 & $\pm 0,27$ & 6,12 \\
Farinha B & 19,01 & $\pm 1,85$ & 9,74 \\
Farinha P & 106,07 & $\pm 2,28$ & 2,15 \\
\hline
\end{tabular}

Fonte: Dados do Estudo

Segundo Campos \& Rosado (2005), o valor de betacaroteno encontrado em cenoura minimamente processada foi de $(1,6 \mathrm{mg} / 100 \mathrm{~g})$. Na farinha de cenoura produzida utilizada na preparação do macarrão - objeto deste estudo - o valor de carotenoides totais segundo análise bromatológica foi de 106,07mg/100g, o que corrobora com o estudo de Campos \& Rosado (2005) no qual afirma que o processamento e a homogeneização mecânica dos alimentos e a consequente redução do tamanho das partículas podem aumentar a biodisponibilidade dos carotenoides. Alguns estudos que analisaram o conteúdo de carotenoides em alimentos, reportaram maior facilidade de extração dos carotenoides, após o processamento térmico, produzindo aumento na quantidade total de carotenoides pró vitamínicos A em relação aos vegetais frescos.

A proporção encontrada de betacaroteno na farinha produzida foi de $21,28 \mathrm{mg} / 100 \mathrm{~g}$. Sabe-se que o RE (equivalente de retinol) para esse carotenoide é dado pela proporção 1RE $=12 \mu \mathrm{g}$ de betacaroteno, $\log 0$ na porção de $50 \mathrm{~g}$ do macarrão obtiveram-se um total de 886,67mg o que corresponde a 115,65\% da recomendação de ingestão diária, porém não atribuindo qualquer risco ao consumidor considerando a UL (nível máximo de ingestão diária) de $2750 \mu \mathrm{g} /$ dia. Apesar da porção definida aparentar ser pequena é a quantidade suficiente para uma refeição, considerando ser esse o peso do alimento cru e com índice de conversão igual a 1,69. Ou seja, a porção final de $50 \mathrm{~g}$ da massa crua, após ser cozida, será de aproximadamente $84,5 \mathrm{~g}$. 


\section{Massa Produzida}

O macarrão é um alimento que possui de alto teor calórico, mas ainda sim se faz constantemente presente na mesa da população por sua praticidade e versatilidade. Pensando nisso, optou-se em desenvolver um produto funcional fonte de pró vitamina A, e com o valor nutricional diferenciado. Para isso, foi produzida uma massa fresca com farinha de cenoura, sendo essa uma das hortaliças mais produzidas e consumidas pela população brasileira (SILVA et al., 2016).

Por fim, a Tabela 2 dispõe sobre as informações nutricionais do macarrão de acordo com Ficha Técnica de preparo realizada no teste de receita.

\section{Tabela 2: Composição centesimal Macarrão enriquecido com farinha de cenoura}

\begin{tabular}{cc}
\hline NUTRIENTE & Macarrão (porção de 84g cozido) \\
\hline Calorias & $167,97 \mathrm{Kcal}$ \\
\hline Carboidratos & $28,80 \mathrm{~g}$ \\
Proteínas & $7,3 \mathrm{~g}$ \\
Lipídios & $2,81 \mathrm{~g}$ \\
\hline Lipídeos saturados & $0,65 \mathrm{~g}$ \\
Colesterol & $88,29 \mathrm{mg}$ \\
Fibras & $4,58 \mathrm{~g}$ \\
\hline Fonte: Dados do Estudo &
\end{tabular}

Os resultados salientam a possibilidade de inserção da massa na dieta da população brasileira já que a mesma atinge aos níveis esperados de betacaroteno contidos na cenoura. 


\section{Conclusão}

Diante dos dados obtidos pela revisão bibliográfica, pode-se destacar a relevância do uso de vitamina A no processo de inflamação da acne, agindo como imunomoduladora no indivíduo, a fim de reduzir a gravidade da inflamação e, consequentemente, redução do número de anticorpos para proteção das células afetadas no local. Os aspectos nutricionais ficam evidentes na farinha de cenoura produzida, já que os resultados da análise bromatológica demonstraram quantidades relevantes se comparados com quantidade de carotenoides totais em farinhas já existentes no mercado.

Com base nas evidências cientificas e os resultados obtidos pela análise bromatológica realizada, observou-se que a quantidade de betacaroteno presente na cenoura é de grande relevância para redução do quadro inflamatório da acne, além de comprovar a presença em maior quantidade do nutriente citado na farinha produzida, justificando-se a produção do macarrão e os resultados positivos encontrados na composição centesimal da massa preparada. Sendo assim, fica evidente o excelente potencial e efetividade da vitamina A na redução da inflamação na acne.

\section{REFERÊNCIAS}

AGAK, G.W. et al. Propionibacterium acnes induces an interleukin-17 response in acne vulgaris that is regulated by vitamin A and vitamin D. The Journal of investigative dermatology. p.366-373, 2014.

\section{AGÊNCIA NACIONAL DE VIGILÂNCIA SANITÁRIA. Resolução RDC n93, de 31 de} dezembro de 2000. Disponível

em:<http://www.anvisa.gov.br/anvisalegis/resol/2000/93_00rdc.htm>. Acessoem: 02 de março de 2018.

BECKENBACH, L. et al. Retinoid treatment of skin diseases.Journal of the European Academy of 
BIESKI, G. L. Riscos e benefícios do uso do medicamento isotretinoína para o tratamento da acne. FACIDER RevistaCientífica, Colider MT, n. 09, 2016.

BORREGAARD, N.; SORENSEN, O. E.; THEILGAARD-MONCH, K.Neutrophil granules: a library of innate immunity proteins. Trends in Immunology. v.28, n.8: p.340345.2007.

CAMPOS, Flávia Milagres; ROSADO, Gilberto Paixão. Novos fatores de conversão de carotenóidesprovitamínico A. Ciências Tecnologia de Alimentação, Campinas, ed. 25, v.3: p. 571- 578, jul.-set. 2005.

CHAPMAN, M.S. Vitamin A: History, Current Uses, and Controversies.Seminars in Cutaneous Medicine and Surgery. Elsevier INC. v.31, p.11-16, 2012.

FIGUEIREDO, A. et al. Avaliação e tratamento do doente com acne - Parte I:

Epidemiologia, etiopatogenia, clínica, classificação, impacto psicossocial, mitos e realidades, diagnóstico diferencial e estudos complementares. Revista Portuguesa de Clínica Geral. v. 27, n. 1: p. 59-65.Lisboa, janeiro, 2011.

INSTITUTO BRASILEIRO DE GEOGRAFIA E ESTATÍSTICA. Pesquisa de Orçamentos Familiares 2008-2009: análise do consumo alimentar pessoal no Brasil. Brasília: IBGE, 2013. Disponível em: < http://www.ibge.gov.br/home/estatistica/populacao/pns/2013/>. Acesso em 24 de fevereiro de 2017.

KUROKAWA, I.New developments in our understanding of acne pathogenesis and treatment. Experimental Dermatology. v.18, p.821-832, 2009.

MEDEIROS, G.R. et al. Avaliação De Carotenóides Em Cenoura E Análise Sensorial De Barras De Cereais Elaboradas Com Cenoura Desidratada. Revista Brasileira de Tecnologia Agroindustrial. v. 05, n. 01, p. 306-313, 2011. 
NAST.A. et al. Guidelines for the Treatment of Acne. Journal of the European Academy of Dermatology and Venereology. p. 1-29, 2012.

RIBEIRO. B et al. Etiopathogeny of acne vulgaris: a practical review for day-to-day dermatologic practice. SurgicalCosmetDermatol, ed.7, p. 6-20, 2015.

RODRIGUEZ-AMAYA, D. et al. Carotenoid pigment changes in ripening Momordicacharantia fruits. Annals of Botany.v.40, p.615-624.1976.

SILVA, Ana Carolina Bizinoto et al. Qualidade nutricional e físico-química em cenoura (Daucuscarotal.) in natura e minimamente processada. Demetra: alimentação, nutrição \& saúde, Uberlandia, Minas Gerais, Brasil. 2016.

TACO- Tabela Brasileira de Composição de Alimentos. ed.4. Campinas NEPA/UNICAMP. p.2011-161, 2011.

TONHL, E. et al. Fases estacionarias para Cromatografia Liquida de Alta Intensidade em fase reversa (CLAE-FR) baseada em superfícies de óxidos orgarnicos funcionalizados.

Quimica Nova, v. 25, n.4: p.616-623, 2002.

ZOUBOULIS, C.C.; JOURDAN, E.; PICARDO, M. Acne is an inflammatory disease and alterations of sebum composition initiate acne lesions. Journal of the European Academy of Dermatology and Venereology.v.28, p.527-532.Maio, 2014. 